\title{
Quick Inspection of Power-Plane Short Fault on Multilayer Substrate
}

\author{
Fang-Lin Chao and Ruey-Beei Wu, Member, IEEE
}

\begin{abstract}
A quick inspection approach for finding the powerplane short fault on multichip modules is proposed in this paper. The current source is applied on two diagonal corners of the power plane. The location of the short fault can be determined by as few as four voltage measurements. Also, the sheet conductivity of the power plane can be obtained as a by-product. Experiments have been performed and the successful prediction of the exact short point validates the present inspection approach.
\end{abstract}

Index Terms - Inspection, multichip module, short fault, conductivity measurement, multilayer substrate.

\section{INTRODUCTION}

$\mathbf{T}$ IOGETHER with the evolution of integrated circuit technology is the ever-increasing device density, switching speed, and system performance [1]. Multilayer structure has become the essential trend of substrate design to increase interconnection density [2], [3]. For high performance multichip modules, the existence of numerous contact points represents greater challenges to fabricate and inspect the substrate. Since inspection facilities and labor cost are increasingly expensive, inspection reduction is important for reducing manufacturing cost.

One of the common faults in multilayer boards or MCM's is the power plane short problem. The different power and ground planes might be shorted due to manufacturing variations, defects in via formation process, etc. Automatic optical inspection (AOI) techniques have been widely utilized in printed circuit board inspection. The circuit pattern on each laminated layer is scanned by a computer-controlled optical beam and the resultant image is compared with a golden sample. Any abnormal patterns located by AOI are then corrected by technicians. However, AOI cannot inspect all the short problems. For example, it cannot detect the defects incurred by vias in the laminating process. Also, MCMD substrates which are formed by a deposition process are difficult to inspect by such an optical technique.

\section{InSPECtion OF POWER-Plane Short Fault}

Two approaches to inspect the power plane short fault are investigated and compared in this study. One is to impose a

Manuscript received November 9, 1994; revised February 2, 1995. This paper was revised from a presentation at the International Electronics Manufacturing Technology Symposium, La Jolla, CA, September 12-14, 1994.

F.-L. Chao was with the Electronic Research and Service Organization, Industrial Technology Research Institute, Hsinchu, Taiwan, ROC. He is now with the Da-Yeh Institute of Technology, Chung-Hwa, Taiwan, ROC.

R.-B. Wu is with the Department of Electrical Engineering, National Taiwan University, Taipei, Taiwan, ROC.

IEEE Log Number 9411281.

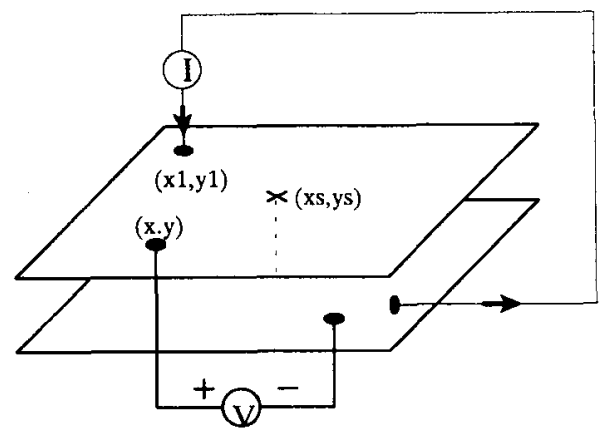

Fig. 1. Intuitive inspection approach by driving current source across power and ground planes.

dc voltage source between the power plane and the ground plane. Fig. 1 illustrates the setup of this intuitive inspection approach. The current flows from the power plane, through the short, and to the ground plane. The short point can be sought by probing voltage distribution on the substrate. One can measure the voltage values at power pads. The lower the value, the closer the pad is to the short point. However, most MCM substrates have only a few positions convenient for voltage measurement. On occasion, none of these available probing points are close to the short location. This makes the inspection difficult, time consuming, and sometimes in vain.

The other approach is to drive the current through the power plane only. Fig. 2 illustrates the setup of this quick inspection approach. The current source is applied between two diagonal corners of the substrate. Due to the finite conductivity of the metal, a potential distribution is established on the power plane. If there is a short point on the substrate, the potential of this point is equal to that of any point in the ground plane. Choosing the ground plane as the voltage reference, the fault will lie in the zero voltage contour line. By changing the current source to another pair of opposite corners, one can determine another associated zero voltage contour line. The intersection of the two zero voltage contour lines locates the position of the short fault.

To be explained later, this approach requires few probing points to determine the zero voltage contour line. It works well even if the short point is not close to any of the probing points. Therefore, the approach is more convenient and successful for inspection of the MCM power plane short faults.

\section{iII. Calculation of Potential Distribution}

Consider the experimental setup shown in Fig. 2 where the power plane is of size $a \times b$, the thickness is $t$, and the conductivity is $\sigma$. Assume that the unknown short fault is 


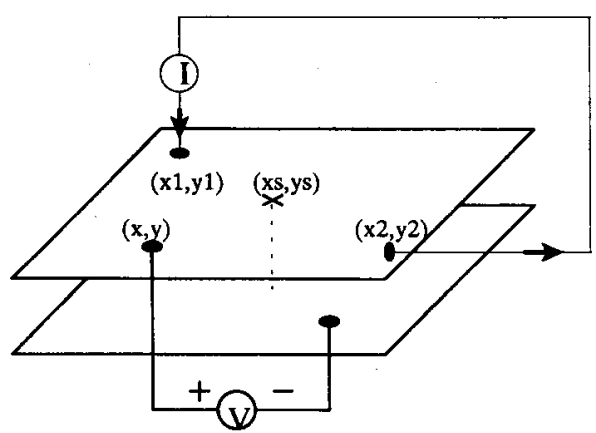

Fig. 2. Quick inspection approach by driving current source across two diagonal comers at power plane.

located at $\left(x_{s}, y_{s}\right)$. A current of amplitude $I$ flows from the driving point $\left(x_{1}, y_{1}\right)$, distributes in the plane, and merges to the sink point $\left(x_{2}, y_{2}\right)$. Choosing the short point as the voltage reference, it can be shown that the voltage distribution $V(x, y)$ in the plane satisfies [4]

$$
\begin{aligned}
& -\left(\frac{\partial^{2} V}{\partial x^{2}}+\frac{\partial^{2} V}{\partial y^{2}}\right) \\
& =\frac{I}{\sigma_{\square}}\left[\delta\left(x-x_{1}\right) \delta\left(y-y_{1}\right)-\delta\left(x-x_{2}\right) \delta\left(y-y_{2}\right)\right]
\end{aligned}
$$

subject to the homogeneous Neumann boundary condition at all the four sides of the plane. Here, $\sigma_{\square}=\sigma \cdot t$ is the sheet conductivity of the power plane.

By applying the eigenfunction expansion method, the voltage distribution is solved in the Appendix and can be written as

$$
V(x, y)=\frac{I}{\sigma_{\square}}\left[\tilde{\phi}(x, y)-\tilde{\phi}\left(x_{s}, y_{s}\right)\right]
$$

where the normalized potential distribution

$$
\tilde{\phi}(x, y)=\sum_{m, n=0}^{\infty} A_{m n} \cos \frac{m \pi x}{a} \cos \frac{n \pi y}{b}
$$

in which $A_{00}=0$,

$$
A_{m n}=\frac{\epsilon_{m} \epsilon_{n}}{a b} \frac{\cos \frac{m \pi x_{1}}{a} \cos \frac{n \pi y_{1}}{b}-\cos \frac{m \pi x_{2}}{a} \cos \frac{n \pi y_{2}}{b}}{\left(\frac{m \pi}{a}\right)^{2}+\left(\frac{n \pi}{b}\right)^{2}}
$$

for $m^{2}+n^{2} \neq 0$, and

$$
\epsilon_{m}=\left\{\begin{array}{ll}
1 & \text { if } m=0 \\
2 & \text { if } m \neq 0
\end{array} .\right.
$$

The expression, though compact in the form, contains a double series which converges very slowly. The Appendix lists two alternate representations which involve only single series of exponentially decaying terms. They are actually employed in the numerical computation.

For the intuitive inspection approach where the current source is across the power and ground planes, the short point serves as the current sink. Fig. 3 shows the potential for the case with $a=45, b=50,\left(x_{1}, y_{1}\right)=(4.8,48)$, and $\left(x_{2}, y_{2}\right)=$ $(19.2,28.8)$. Owing to the higher current density at the short point, the potential gradient there is much greater than that in other region. It means that the short point is located in the maximum voltage gradient region. In most of the other region, the voltage changes comparatively slightly. This makes the inspection search even more difficult since one must try

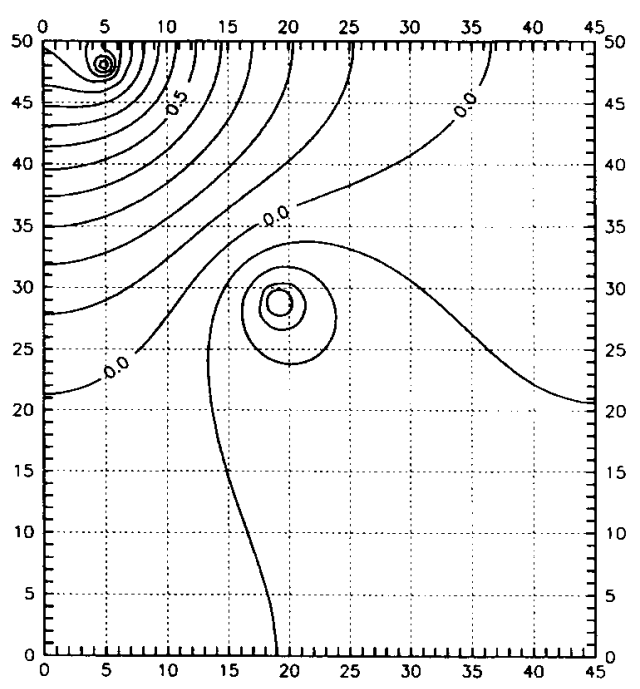

Fig. 3. Equipotential contour map in intuitive inspection approach.

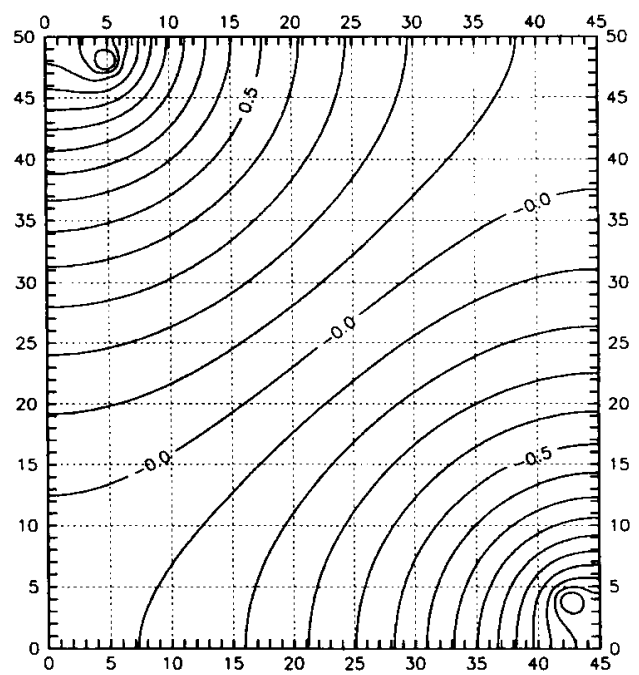

Fig. 4. Equipotential contour map in the quick inspection approach.

the voltage measurements at many points to decide the right direction of the next trial.

Secondly, the quick inspection approach with current driving across two opposite corners in the power plane is analyzed. Fig. 4 shows the equipotential contour map for the case with $\left(x_{1}, y_{1}\right)=(4.8,48)$ and $\left(x_{2}, y_{2}\right)=(42.5,3.8)$. The maximum gradient positions happen at the driving and sinking points in the power plane. The voltage gradient at the short point is comparatively much smaller. Few voltage measurements (at least two) are sufficient to identify which equipotential contour line corresponds to the zero voltage line. The short point must lie somewhere in that line. Imposing current at different points can provide additional zero voltage line to uniquely locate the desired short fault from their intersection.

\section{EXPERIMENT}

A test board is built to validate the inspection approach. For simplicity of the measurement, the test board is enlarged to 45 $\mathrm{cm} \times 50 \mathrm{~cm}$ in size. A short via between power and ground planes is artificially made at the indicated point $(19.2,28.8)$. In the first setup, the current source is applied to the two diagonal 
TABLE I

MEASuRed Voltage

\begin{tabular}{r|r|r|r|r|r}
\hline & & \multicolumn{2}{|c|}{ case (a) } & \multicolumn{2}{c}{ case (b) } \\
\cline { 3 - 6 }$x$ & $y$ & $V(x, y)$ & $\phi(x, y)$ & $V(x, y)$ & $\phi(x, y)$ \\
\hline 8.6 & 7.0 & -0.65 & -0.0863 & 2.14 & 0.7707 \\
\hline 8.6 & 16.2 & -0.36 & 0.0161 & - & - \\
\hline 8.6 & 19.2 & -0.22 & 0.0657 & 0.90 & 0.3310 \\
\hline 8.6 & 29.9 & 0.45 & 0.3054 & 0.21 & 0.0823 \\
\hline 8.6 & 12.0 & 1.54 & 0.7251 & -0.23 & -0.0790 \\
\hline 16.0 & 10.0 & -0.77 & -0.1460 & 1.26 & 0.4310 \\
\hline 16.0 & 19.1 & -0.39 & -0.0161 & 0.66 & 0.2247 \\
\hline 16.0 & 26.8 & 0.04 & -0.1342 & 0.19 & 0.0606 \\
\hline 16.0 & 39.0 & 0.79 & 0.4090 & -0.34 & -0.1352 \\
\hline 28.0 & 14.7 & -1.06 & -0.3015 & 0.45 & 0.1048 \\
\hline 28.0 & 23.8 & -0.53 & -0.1082 & -0.01 & -0.0586 \\
\hline 28.0 & 37.5 & 0.14 & 0.1361 & -0.78 & 0.3461 \\
\hline 38.8 & 5.6 & -2.58 & -0.9426 & 0.43 & 0.0819 \\
\hline 38.8 & 16.2 & -1.44 & -0.4704 & 0.12 & -0.0328 \\
\hline 38.8 & 29.9 & -0.48 & -0.1026 & -0.70 & -0.3410 \\
\hline 38.8 & 40.5 & -0.05 & 0.0516 & -1.73 & -0.7482 \\
\hline
\end{tabular}

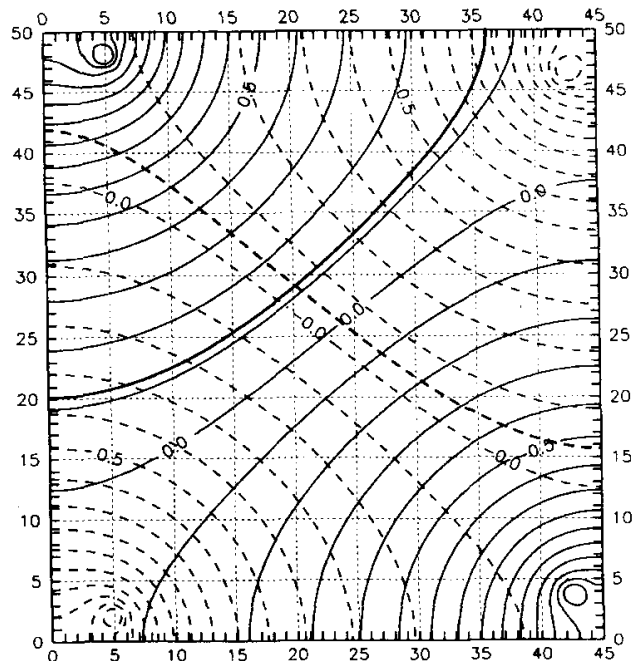

Fig. 5. Determination of short fault point from intersection of two zero voltage contour lines.

corners at $(4.8,48)$ and $(42.5,3.8)$. The equipotential contour map of the normalized potential distribution $\tilde{\phi}(x, y)$ is shown in Fig. 4. By moving the voltage meter across the board, the measured voltages $V(x, y)$ on the plane are listed in case (a) of Table I. Given that $V(x, y)$ is linearly related to $\tilde{\phi}(x, y)$ as depicted by (2), the linear regression can be employed to find the best fit function

$$
V(x, y)=-0.2966+2.493 \tilde{\phi}(x, y) .
$$

Hence, the equipotential line where the short fault lies is the one with $\tilde{\phi}\left(x_{s}, y_{s}\right)=0.1180$. It is plotted in the equipotential contour map as a broad solid curve in Fig. 5.

In the second setup, the current source is applied to the other diagonal corners at $(4.8,2.2)$ and $(42.5,46.5)$. The equipotential contour of the normalized potential distribution $\tilde{\phi}(x, y)$ for this case can be obtained and is drawn as the dashed lines in Fig. 5. The case (b) in Table I lists the values of $\tilde{\phi}(x, y)$ and the measured $V(x, y)$ at same probing points. For the present, the best fit function is

$$
V(x, y)=0.1125+2.552 \tilde{\phi}(x, y)
$$

determined by linear regression. The short fault lies in the equipotential line with $\hat{\phi}\left(x_{s}, y_{s}\right)=-0.0435$, which is plotted as a broad dashed curve in Fig. 5.
Finding the intersection of the two equipotential lines obtained in the two setups yields the short point $\left(x_{s}, y_{s}\right)=(20.1$, 29.1). The excellent agreement with the exact short point (19.2, 28.8) validates the present inspection approach.

It is worthy mentioning that the sheet conductivity of the power plane can be obtained in the meantime as a by-product. The slope of the best fit linear function should equal $\frac{I}{\sigma}$ as depicted by (2). Given the measured current ( $I=3 \mathrm{~A})$, the sheet conductivity can be determined to be

$$
\sigma_{\square} \cong \frac{1}{2}\left(\frac{3}{2.493}+\frac{3}{2.552}\right)=1.19 \mathrm{~K} \text { mho. }
$$

Since the power plane is made of copper, its thickness will be

$$
t=\frac{\sigma_{\square}}{\sigma}=20.9 \mu \mathrm{m} \text {. }
$$

\section{CONCLUSION}

A power plane short fault can be created by mask misalignment, manufacturing variation, or defects in via formation process. In this study, current source is applied on two diagonal corners of the power plane. The equipotential contour line where the short fault lies can be found by the voltage measurement of as few as two probing points. Applying the current source to another two corners can find another equipotential contour and then determine the short point. In the meantime, the sheet conductivity of the power plane can be obtained accurately. Analytical formula has been derived to give the potential distribution for a plane with a rectangular shape. A test board was built and the short fault was successfully located. It demonstrates that this inspection approach is quite helpful in MCM repair/rework.

Although demonstrated for the rectangular power plane only, the present quick inspection approach works as well for power planes of other shapes or even perforations. The analytical formula for the normalized potential distribution becomes almost impossible. Nonetheless, one can employ the finite element method to obtain the equipotential contour map numerically. Then, the location of the short fault can be determined by a similar procedure.

\section{APPENDIX \\ DERIVATION OF NORMALIZED POTENTIAL Distribution}

The normalized potential distribution $\tilde{\phi}(x, y)$ defined in this paper satisfies the partial differential equation

$$
\begin{aligned}
& -\left(\frac{\partial^{2} \tilde{\phi}}{\partial x^{2}}+\frac{\partial^{2} \tilde{\phi}}{\partial y^{2}}\right) \\
& =\delta\left(x-x_{1}\right) \delta\left(y-y_{1}\right)-\delta\left(x-x_{2}\right) \delta\left(y-y_{2}\right)
\end{aligned}
$$

in the region $0 \leq x \leq a$ and $0 \leq y \leq b$ subject to the homogeneous Neumann boundary conditions at the four sides. It is easily verified that the solution to (A1) is not unique since $\tilde{\phi}+c$ where $c$ is an arbitrary constant can satisfy (A1) too. For this sake, it is conventional to choose the one having zero mean, i.e.,

$$
\int_{0}^{a} \int_{0}^{b} \tilde{\phi}(x, y) d y d x=0
$$


The associated Green's function $G\left(x, y ; x^{\prime}, y^{\prime}\right)$ is governed by

$$
\begin{aligned}
-\nabla^{2} G & \equiv-\left(\frac{\partial^{2} G}{\partial x^{2}}+\frac{\partial^{2} G}{\partial y^{2}}\right) \\
& =\delta\left(x-x^{\prime}\right) \delta\left(y-y^{\prime}\right)-\frac{1}{a b}
\end{aligned}
$$

subject to the same Neumann boundary conditions at the four sides and the zero mean condition defined in (A2). Here, the last constant term in (A3) which nullifies the mean of the righthand side is necessary to make the equation solvable. Once the Green's function can be obtained, the desired solution to (A1) can be written as

$$
\tilde{\phi}(x, y)=G\left(x, y ; x_{1}, y_{1}\right)-G\left(x, y ; x_{2}, y_{2}\right) .
$$

Several representations for the solution of (A3) exist. The most common one is obtained by the eigenfunction expansion. The solution is written in the form

$$
G(x, y)=\sum_{m=0}^{\infty} \sum_{n=0}^{\infty} G_{m n} \cos \frac{m \pi x}{a} \cos \frac{n \pi y}{b} .
$$

The coefficient $G_{00}=0$, due to the zero mean condition and the other coefficients

$$
G_{m n}=\frac{\epsilon_{m} \epsilon_{n}}{a b} \int_{0}^{a} \int_{0}^{b} G(x, y) \cos \frac{m \pi x}{a} \cos \frac{n \pi y}{b} d y d x
$$

where $\epsilon_{m}=1$ for $m=0$ while $\epsilon_{m}=2$ for $m \neq 0$, and similarly for $\epsilon_{n}$.

The coefficient $G_{m n}$ can be solved by taking inner product between the eigenfunction

$$
\psi_{m n}(x, y)=\cos \frac{m \pi x}{a} \cos \frac{n \pi x}{b}
$$

and (A3). Using the Green's identity

$$
\begin{aligned}
& -\int_{0}^{a} \int_{0}^{b} G(x, y) \nabla^{2} \psi_{m n}(x, y) d y d x \\
& =-\int_{0}^{a} \int_{0}^{b} \psi_{m n}(x, y) \nabla^{2} G(x, y) d y d x
\end{aligned}
$$

one can obtain

$$
\left[\left(\frac{m \pi x}{a}\right)^{2}+\left(\frac{n \pi y}{b}\right)^{2}\right] G_{m n}=\cos \frac{m \pi x^{\prime}}{a} \cos \frac{n \pi y^{\prime}}{b}
$$

for $m^{2}+n^{2} \neq 0$. Substitution of the coefficients $G_{m n}$ found from (A8) to (A5) yields the eigenfunction expansion solution of the Green's function. However, the expansion includes a double series and converges very slowly.

To facilitate the numerical computation, other representations of the Green's function can be sought for. For example, one can expand the Green's function in terms of the basis functions in $x$-direction and end up with the expression

$$
G(x, y)=\sum_{m=0}^{\infty} G_{m}(y) \cos \frac{m \pi x}{a}
$$

where the coefficient function

$$
G_{m}(y)=\frac{\epsilon_{m}}{a} \int_{0}^{a} G(x, y) \cos \frac{m \pi x}{a} d x .
$$

Taking the inner product of (A3) with these basis functions and applying integration by part, the function $G_{m}(y)$ can be shown to satisfy

$$
-\frac{d^{2} G_{0}}{d y^{2}}=\frac{1}{a}\left[\delta\left(y-y^{\prime}\right)-\frac{1}{b}\right]
$$

subject to the zero mean condition for $m=0$ and

$$
-\left[\frac{d^{2}}{d y^{2}}-\left(\frac{m \pi}{a}\right)^{2}\right] G_{m}=\frac{2}{a} \delta\left(y-y^{\prime}\right) \cos \frac{m \pi x^{\prime}}{a}
$$

for $m \neq 0$, both subject to the homogeneous Neumann boundary condition at $y=0$ and $b$.

It is a typical technique, though quite tedious, in mathematical physics to solve (A11) and (A12) [5]. The solutions can be found to be

$$
G_{0}(y)=\frac{y_{<}^{2}+\left(b-y_{>}\right)^{2}}{2 a b}-\frac{b}{6 a}
$$

where $y_{<} \equiv \min \left(y, y^{\prime}\right), y_{>} \equiv \max \left(y, y^{\prime}\right)$, and

$$
G_{m}(y)=\frac{2 \cos \frac{m \pi x^{\prime}}{a} \cosh \frac{m \pi y_{<}}{a} \cosh \frac{m \pi\left(b-y_{>}\right)}{a}}{m \pi \sinh \frac{m \pi b}{a}}
$$

for $m \neq 0$. Substituting (A13) and (A14) into (A9) yields an alternate representation to the Green's function. This expression is a single series with its terms exponentially decaying by the factor $\exp \left(-\frac{m \pi\left|y-y^{\prime}\right|}{a}\right)$ for large $m$. Hence, the representation is very efficient for numerical computation except when $y$ is very close to $y^{\prime}$.

Even in case that $y \cong y^{\prime}$, one can employ another expression for efficient numerical computation. Following a similar derivation but now expanding the Green's function in terms of the basis functions in $y$-direction, one can have another representation, i.e.,

$$
\begin{aligned}
G(x, y)= & \sum_{n=1}^{\infty} \frac{2 \cosh \frac{n \pi x_{<}}{b} \cosh \frac{n \pi\left(a-x_{>}\right)}{b}}{n \pi \sinh \frac{n \pi a}{b}} \\
& \times \cos \frac{n \pi y^{\prime}}{b} \cos \frac{n \pi y}{b}+\frac{x_{<}^{2}+\left(a-x_{>}\right)^{2}}{2 a b}-\frac{a}{6 b} .
\end{aligned}
$$

The terms in (A15) are exponentially decaying by the factor $\exp \left(-\frac{n \pi\left|x-x^{\prime}\right|}{b}\right)$ for large $n$. This representation can be used to evaluate $G(x, y)$ very efficiently if only $x$ is not very close to $x^{\prime}$.

\section{REFERENCES}

[1] E. E. Davidson and G. A. Katopis, "Package electrical design," in Microelectronic Packaging Handbook, R. R. Tummala and E. J. Rymaszewski Eds. New York: Van Norstrand Reinhold, 1989, chap. 3.

[2] A. J. Blodgett, Jr., "Microelectronic packaging," Scientific American, vol. 249, pp. 86-96, July 1983.

[3] T. Dixon, "Multilayer ceramics: The key to high density interconnections," Electronic Packaging \& Production, pp. 76-82, Feb. 1983.

[4] R. B. Wu, "Resistance computations for multi-layer packaging structure by boundary element method," IEEE Trans. Comp., Hybrids, Manufact. Technol., vol. 15, pp. 87-96, Jan. 1992.

[5] B. Friedman, Principles and Techniques of Applied Mathematics, 1958, chap. 3 . 


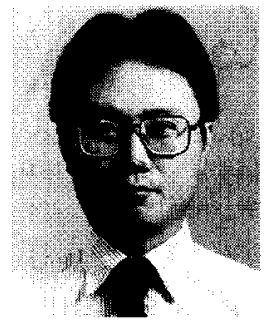

Fang-Lin Chao was born in Taipei, Taiwan, ROC, in 1958. He received the B.S. degree from ChungYuan University, China, in 1980, and the M.S. degree from National Central University, China, in 1982, both in physics, and the Ph.D. degree in electrical engineering from National Taiwan University, ROC, in 1991.

He joined the Electronic Research and Service Organization, Industrial Technology Research Institute, Hsinchu, Taiwan, ROC, in 1982. His responsibilities included thermal, structural, and electrical modeling for the system, PCB, and MCM. Since September 1994, he has been an Associate Professor with the Department of Industrial Design at Da-Yeh Institute of Technology, Chung-Hwa, Taiwan, ROC. His research interests include electronic packaging electrical design, electromagnetic compatibility, and design integration in product development.

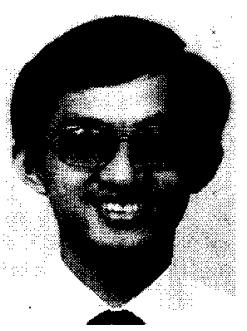

Ruey-Beei Wu (M'91) was born in Tainan, Taiwan, ROC, in 1957. He received the B.S.E.E. and Ph.D degrees from National Taiwan University, Taipei, Taiwan, ROC, in 1979 and 1985, respectively.

In 1982, he joined the faculty of the Department of Electrical Engineering, National Taiwan University, where he is now a Professor. He was a visiting scholar in IBM East Fishkill Facility, NY, from 1986 to 1987, and in the Electrical Engineering Department, University of California, Los Angeles, from August 1994 to July 1995. His areas of interest include computational electromagnetics, dielectric waveguides, edge slot antennas, wave scattering of composite materials, transmission line discontinuities, and interconnection modeling for computer packaging. 